Bangladesh J. Plant Taxon. 12(2): 49-62, 2005 (December)

\title{
HYDROBIOLOGICAL STUDIES WITHIN THE TEA GARDENS AT SRIMANGAL, BANGLADESH. IV. DESMIDS (17 GENERA)
}

\author{
A. K. M. Nurul Islam* and Haseeb Md. Irfanullah ${ }^{1}$ \\ Department of Botany, University of Dhaka, Dhaka-1000, Bangladesh
}

Key words: Desmids, acidic habitats, phytoplankton, new records

\begin{abstract}
Fifty nine taxa of desmids under 17 genera, namely Cylindrocystis, Netrium, Spirotaenia, Gonatozygon, Penium, Closterium, Pleurotaenium, Triploceras, Triplastrum, Hyalotheca, Groenbladia, Bambusina, Teilingia, Sphaerozosma, Spondylosium, Onychonema and Desmidium have been recorded from different aquatic habitats located within the tea gardens at Srimangal, Maulvi Bazar. Eight taxa are described as new records for Bangladesh including two genera, namely, Cylindrocystis and Spirotaenia.
\end{abstract}

\section{Introduction}

Recently, Islam and Irfanullah have described the aquatic macrophytes (Islam and Irfanullah 2000) and algae (excluding desmids) (Islam and Irfanullah 2005a, 2005b) of some selected habitats within the tea gardens at Srimangal, Maulvi Bazar. The present paper is the third installment of the same series and it records 17 desmid genera.

\section{Materials and Methods}

For the description and meteorological data of the study area see Islam and Irfanullah (2000). The studied waterbodies, namely, Baraoora Lake, the Burburia River, ditches and paddy fields were mostly acidic (Islam and Irfanullah 2005). In the winter of 1996 (9 January) and in different seasons of 1997 (winter, 6 January; spring, 18 March; rainy season, 20 July and autumn, 20 October), a total of 120 algal samples were collected. See Islam and Irfanullah (2005) for the sample collection methods, and their preservation and examination.

\section{Taxonomic enumeration}

The present study reveals 59 taxa of desmids belonging to 17 genera of which eight taxa are newly recorded for Bangladesh (marked by* asterisks). Nonetheless, a few algal taxa have already been reported as new records by the same authors from this area (Islam and Irfanullah 1998, 1999), which are not marked in this account.

*Corresponding author. ${ }^{1}$ IUCN - The World Conservation Union, Bangladesh Country Office, House 11, Road 138, Gulshan 1, Dhaka 1212. E-mail: hmirfanullah@yahoo.co.uk 


\section{Class: Chlorophyceae; Order: Zygnematales; Family: Mesotaeniaceae Genus: Cylindrocystis Meneghini ex Ralfs 1848}

1. *? C. brebissonii Meneghini

(P1. 3, Fig. 30)

(Scott and Prescott 1961, 1:3)

L. $47.2 \mu \mathrm{m}$, W. $23 \mu \mathrm{m}$, t. 10.8-13.5 $\mu \mathrm{m}$; two pyrenoids per cell. River; spring 1997; few.

\section{Genus: Gonatozygon de Bary, 1858}

2. G. aculeatum Hastings

(P1. 5, Fig. 48) (Smith 1924, 52: 3; Scott and Prescott 1961, 1: 7)

L. $250 \mu \mathrm{m}, \mathrm{W}$. csp. $20-21.6 \mu \mathrm{m}$, W. ssp. $10.8-12 \mu \mathrm{m}$, t. ssp. 12-12.8 $\mu \mathrm{m}$. Lake; winter 1997; common.

3. G. kinahani (Archer) Rab. fa. (Islam and Irfanullah 1999, 118, 1: 4)

Lake; autumn 1997; few.

4. G. pilosum Wolle (Růžička 1977, 1: 15-16)

(P1. 5, Fig. 49)

L. $262 \mu \mathrm{m}, \mathrm{W}$. csp. $13.5 \mu \mathrm{m}$, W. ssp. $12 \mu \mathrm{m}$, t. $12 \mu \mathrm{m}$. Lake; winter 1997; few.

Genus: Netrium (Näg.) Itz. \& Rothe in Rab., 1856

5. N. digitus (Ehr. ex Ralfs) Itz. \& Rothe var. lamellosum (Bréb.) Grönbl. (Islam and Haroon 1980, 2: 27)

(P1. 5, Figs. 50-51)

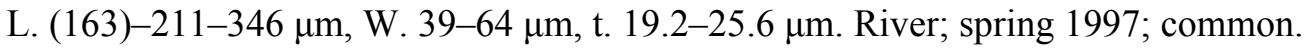

Family: Desmidiaceae; Genus: Spirotaenia de Brébisson, 1844

6. *Spirotaenia sp.

(P1. 3, Fig. 31) (Smith 1924, 5)

L. 86.4-143 $\mu \mathrm{m}, \mathrm{W}$. 13.5-16.2 $\mu \mathrm{m}$; smooth cell wall; spiral chloroplast with pyrenoids. River; spring 1997; common.

Genus: Penium Bréb. ex Ralfs in Ralfs, 1848

7. P. spirostriolatum Barker

(P1. 3, Fig. 32) (Scott and Prescott 1961, 1: 12)

L. $198 \mu \mathrm{m}$, W. $23 \mu \mathrm{m}$, I. $20.2 \mu \mathrm{m}$, t. $13.5 \mu \mathrm{m}$. Lake; winter 1997; rare.

Genus: Triplastrum Iyengar \& Ramanathan, 1942

8. T. abbreviatum (Turner) Iyengar \& Ramanathan

(Pl. 1, Fig. 6) (Islam 1980, 3: 36-41)

L. csp. $85.7 \mu \mathrm{m}$, L. ssp. $83.7 \mu \mathrm{m}, \mathrm{W} .10 .8 \mu \mathrm{m}$, I. $8.5 \mu \mathrm{m}$, t. 11.5-13.5 $\mu \mathrm{m}$. Lake; winter 1996; rare. 


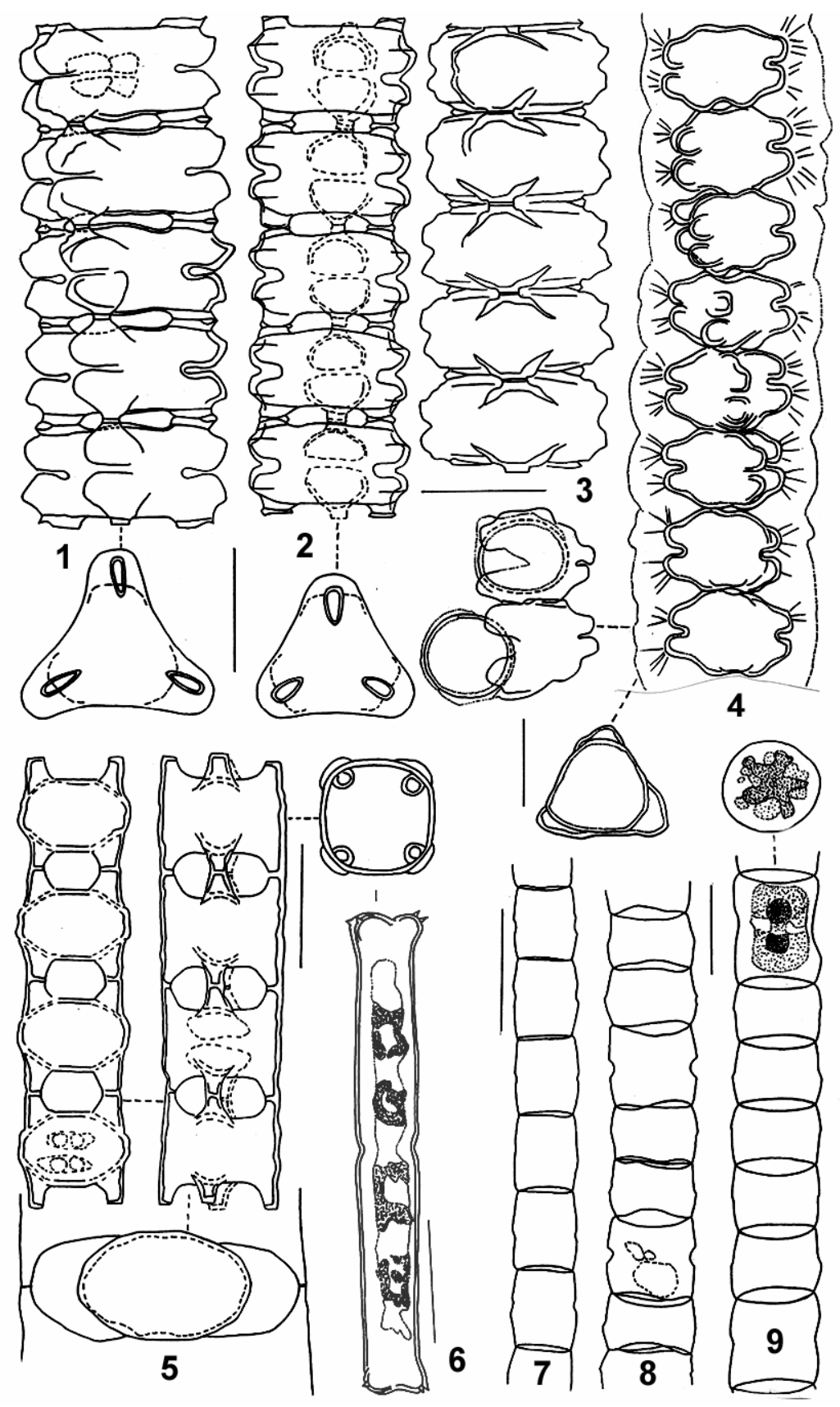

Plate 1

Figs. 1-2. Desmidium aptogonum var. acutius, 3. D. swartzii var. ambloydon, 4. D. bengalicum, 5. D. baileyi var. baileyi, 6. Triplastrum abbreviatum, 7, 9. Hyalotheca dissiliens var. tatrica, 8. H. dissiliens var. hians. [Scales $=20 \mu \mathrm{m}]$ 
Genus: Triploceras Bailey, 1851

9. T. gracile Bailey var. undulatum Scott \& Prescott

(Pl. 5, Fig. 47) (Scott and Prescott 1958, 27, 3: 8)

L. csp. $624 \mu \mathrm{m}$, L. ssp. $608 \mu \mathrm{m}$, W. csp. $52 \mu \mathrm{m}$, W. ssp. $35 \mu \mathrm{m}$, I. $30 \mu \mathrm{m}$, t csp. 46 $\mu \mathrm{m}$, t. ssp. $40.5 \mu \mathrm{m}$. Lake (autumn 1997) and river (spring 1997); few.

Genus: Closterium Nitzsch ex Ralfs, 1848

10. Cl. angustatum Kütz. ex Ralfs

(P1. 2, Fig. 11)

(Islam 1970, 909, 4: 17; Islam and Haroon 1980, 558, 1: 14)

L. $192 \mu \mathrm{m}, \mathrm{W} .13 .5 \mu \mathrm{m}$, t. $6.7-8 \mu \mathrm{m}$. Lake; winter 1997; rare.

11. *Cl. closterioides (Ralfs) Louis and Peeters var. intermedium (Roy \& Biss.) Růžičk (Růžička 1977, 93, 6: 3-6)

(P1. 2, Fig. 18)

L. $109.3 \mu \mathrm{m}$, W. $23 \mu \mathrm{m}$, t. $8-9 \mu \mathrm{m}$; eight axial chloroplasts, smooth wall, pyrenoids were not clearly evident. Lake; winter 1996 and 1997; rare to few.

12. *Cl. dianae var. minus Hieron.

(P1. 2, Fig. 13) (Růžička 1977, 135, 13: 10-12; as C. dianae var. minor Hieron. in Prescott et al. 1975, 47, 23: 8)

L. $213 \mu \mathrm{m}, \mathrm{W} .23 \mu \mathrm{m}$, t. $4.7 \mu \mathrm{m}$. Lake; winter 1997; rare.

13. Cl. gracile Bréb. ex Ralfs

(Prescott et al. 1975, 52, 16: 2; Růžička 1977, 168, 21: 1-4)

(P1. 2, Fig. 12)

L. $416 \mu \mathrm{m}, \mathrm{W} .17 .5 \mu \mathrm{m}$, t. $5.4 \mu \mathrm{m}$; cell wall punctate. Lake; winter 1997; rare.

14. Cl. jenneri Ralfs var. tenue Croasdale

(Pl. 2, Fig. 15)

(Prescott et al. 1975, 56, 23: 2)

L. 97-103 $\mu \mathrm{m}$, W. 9.4-12 $\mu \mathrm{m}$, t. $4 \mu \mathrm{m}$. Lake (winter 1997; few) and river (spring 1997; common).

15. Cl. kuetzingii Bréb. var. vittatum Nordst.

(P1. 2, Fig. 21)

(Růžička 1977, 209, 30: 15-17)

L. $384 \mu \mathrm{m}, \mathrm{W} .13 .5 \mu \mathrm{m}$, t. $2.7 \mu \mathrm{m}$. Lake; winter 1996 and 1997; few.

16. Cl. moniliferum (Bory) Ehr. ex Ralfs

(P1. 2, Fig. 14)

(Prescott et al. 1975, 70, 21: 3)

L. $115 \mu \mathrm{m}$, W. $16.2 \mu \mathrm{m}$, t. $4 \mu \mathrm{m}$. Lake; winter 1996 and 1997, and autumn 1997; few.

17. Cl. pritchardianum Archer

(P1. 2, Fig. 10)

(Prescott et al. 1975, 77, 25: 7, 14)

L. $528 \mu \mathrm{m}$, W. $35.2 \mu \mathrm{m}$, t. $8 \mu \mathrm{m}$. Lake; winter 1997; rare. 


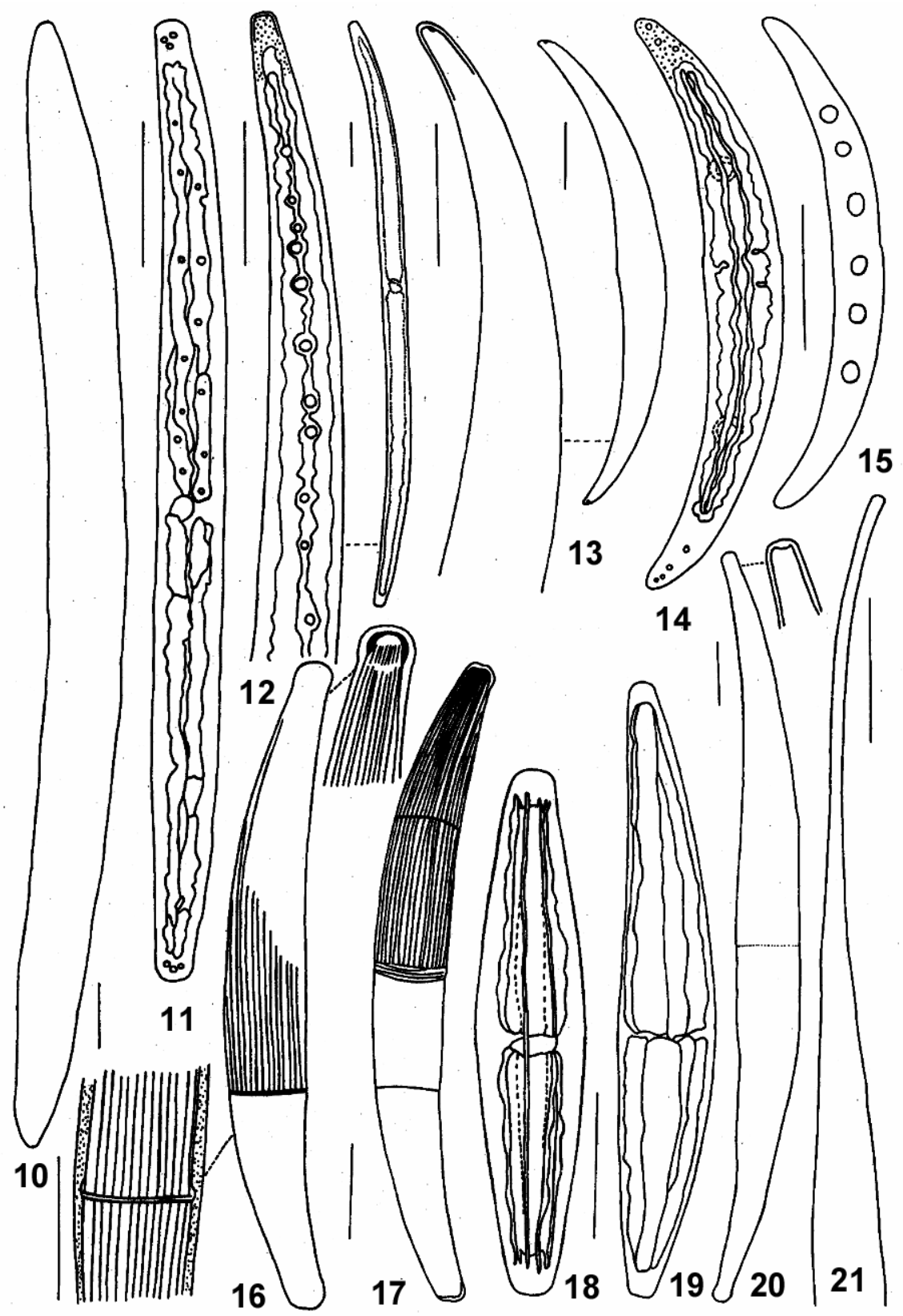

Plate 2

Figs. 10. Closterium pritchardianum, 11. Cl. angustatum, 12. Cl. gracile, 13. Cl. dianae var. minus, 14. Cl. moniliferum, 15. Cl. jenneri var. tenue, 16-17. Cl. striolatum var. subtruncatum, 18. Cl. closterioides var. intermedium, 19. Cl. tumidum, 20. Cl. ralfsii var. hybridum, 21. Cl. kuetzingii var. vittatum. [Scales = $30 \mu \mathrm{m}]$. 
18. Cl. ralfsii de Bréb. var. gracilius (Maskell) Krieger

(Pl. 5, Fig. 58) (Růžička 1977, 191, 25: 9)

L. $143 \mu \mathrm{m}$, W. $5.4 \mu \mathrm{m}$, t. $2 \mu \mathrm{m}$. Lake; winter 1997; few.

19. Cl. ralfsii var. hybridum Rab.

(Pl. 2, Fig. 20) (Růžička 1977, 192, 25: 10-13)

L. $352 \mu \mathrm{m}$, W. $28.8 \mu \mathrm{m}$, t. $6.7 \mu \mathrm{m}$. River; spring 1997; few.

20. * Cl. rectimarginatum Scott \& Prescott

(Pl. 5, Fig. 57) (Scott and Prescott 1961, 13, 1:27-28)

L. $189 \mu \mathrm{m}$, W. $23 \mu \mathrm{m}$, t. $4 \mu \mathrm{m}$. River; spring 1997; rare. Note: it is somewhat similar to C. subfusiforme (see Prescott et al. 1975, 14: 11).

21. Cl. rostratum Ehr. var. rostratum

(P1. 5, Fig. 59)

(Prescott et al. 1975, 83, 31: 3, 12)

L. $243 \mu \mathrm{m}$, W. $20.2 \mu \mathrm{m}$, t. $5.4 \mu \mathrm{m}$. Lake; winter 1996; rare.

22. *Cl. striolatum var. subtruncatum (W. \& W.) Krieger

(Prescott et al. 1975, 89, 27: 5 \& 28: 7; Růžička 1977, 218, 32: 12-14)

L. $192-195 \mu \mathrm{m}$, W. 21.6-24.3 $\mu \mathrm{m}$, t. 9.4-12.2 $\mu \mathrm{m}$; 5-8 striations per $10 \mu \mathrm{m}$. Lake (autumn 1997) and river (spring 1997); few.

23. Cl. tumidum Johnson

(Islam and Akter 1999, 26, 2: 27)

(Pl. 2, Fig. 19)

L. $127 \mu \mathrm{m}$, W. $21.6 \mu \mathrm{m}$, t. $5.4 \mu \mathrm{m}$; six axial chloroplasts per semicell. Paddy field; autumn 1997; few.

Genus: Pleurotaenium Nägeli, 1849

24. Pl. ehrenbergii (Bréb.) de Bary var. ehrenbergii

(Pl. 4, Fig. 37) (Prescott et al. 1975, 114, 45: 1-5)

L. $230 \mu \mathrm{m}, \mathrm{W} .21 .6 \mu \mathrm{m}$, I. $14.8 \mu \mathrm{m}$, t. $13.5 \mu \mathrm{m}$. River; spring 1997; rare.

25. Pl. ehrenbergii var. elongatum West

(P1. 4, Fig. 40)

(Prescott et al. 1975, 117, 46: 1-3)

L. $270 \mu \mathrm{m}, \mathrm{W} .12-13.5 \mu \mathrm{m}$, I. $9.4 \mu \mathrm{m}$, t. 9.4-10 $\mu \mathrm{m}$. Lake; winter 1996; rare.

26. Pl. ehrenbergii var. undulatum Schaars.

(Pl. 4, Fig. 41)

(Islam 1970, 913, 4: 15; Prescott et al. 1975, 117, 46: 5, 6, 18)

L. $512 \mu \mathrm{m}$, W. $54.4 \mu \mathrm{m}$, t. 41.6-43.2 $\mu \mathrm{m}$. Paddy field; autumn 1997; rare.

27. Pl. kayei (Archer) Rab.

(P1. 4, Figs. 42-43)

(Islam and Haroon 1980, 562, 4: 54-55)

L. 275-320 $\mu \mathrm{m}$, W. csp. 56.7-67.5 $\mu \mathrm{m}$, W. ssp. 44.5-54 $\mu \mathrm{m}, \mathrm{I} .32 .4 \mu \mathrm{m}$, t. csp. 38$40.5 \mu \mathrm{m}$, t. ssp. $28 \mu \mathrm{m}$. Lake (autumn 1997; few) and river (winter 1997; common). 


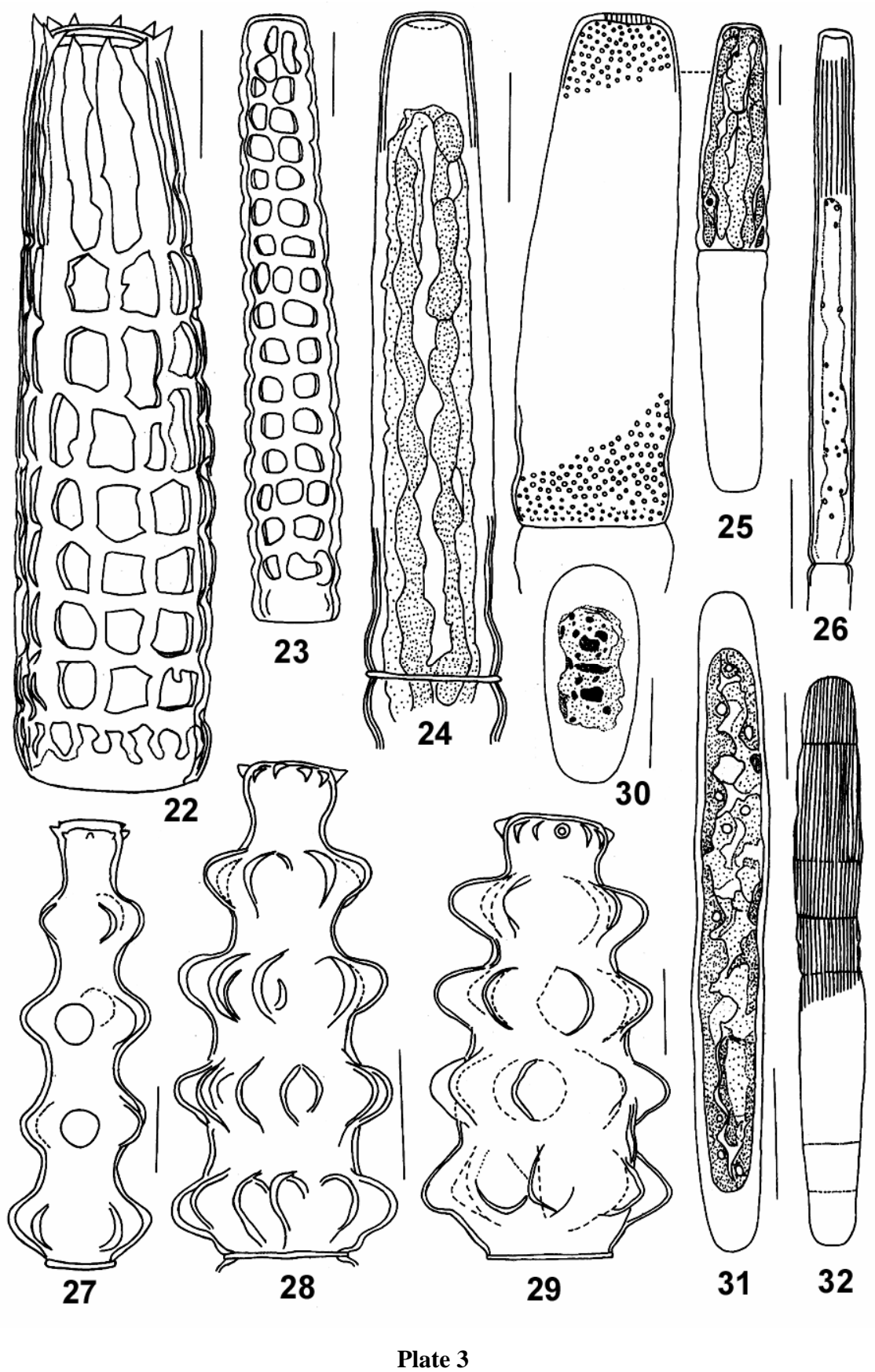

Figs. 22. Pleurotaenium verrucosum var. validum, 23. Pl. trochiscum, 24. Pl. trabecula fa., 25. Pl. trabecula var. crassum, 26. Pl. trabecula var. elongatum, 27. Pl. nodosum var. borgei, 28-29. Pl. nodosum var. gutwinskii, 30. ? Cylindrocystis brebissonii, 31. Spirotaenia sp., 32. Penium spirostriolatum [Scales $=$ $20 \mu \mathrm{m}]$. 
28. Pl. kayei var. ovoideum Islam and Haroon (Islam and Haroon 1980, 562, 5: 77)

L. $243 \mu \mathrm{m}$, W. csp. $71.5 \mu \mathrm{m}$, W. ssp. $56.7 \mu \mathrm{m}$, I. $37.8 \mu \mathrm{m}$, t. csp. $40.5 \mu \mathrm{m}$, t. ssp. 25.6 $\mu \mathrm{m}$. Paddy field; autumn 1997; rare.

29. Pl. nodosum (Bail.) Lund. var. borgei (Grönbl.) Krieger

(P1. 3, Fig. 27) (Islam 1970, 914, 5: 15; Prescott et al. 1975, 125, 44: 6-9)

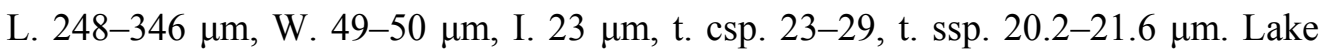
(winter 1997; few) and paddy field (autumn 1997; rare).

30. Pl. nodosum var. gutwinskii Krieger

(P1. 3, Figs. 28-29) (Prescott et al. 1975, 126, 44: 10; Islam and Haroon 1980, 562, 5: 86)

L. 232-313 $\mu \mathrm{m}$, W. 51-86 $\mu \mathrm{m}$, I. $23-43 \mu \mathrm{m}$, t. csp. $24-41 \mu \mathrm{m}$, t. ssp. $22-34 \mu \mathrm{m}$; band present at the isthmus, 10-12 nodules on the basal ring, 12 spines at each semi-cell tip. Quite robust and slightly differs from the typical. Paddy field; autumn 1997; rare.

31. Pl. subcoronulatum (Turn.) W. \& W.

(Scott and Prescott 1961, 132, 49: 2-4, 10; Islam 1970, 914, 4: 1-2)

L. $740 \mu \mathrm{m}$, W. $47 \mu \mathrm{m}$, I. $41 \mu \mathrm{m}$, t. $39 \mu \mathrm{m}$; cell wall pitted. Lake; winter 1996; few.

32. Pl. trabecula (Ehr.) Näg.

(P1. 4, Figs. 33-34)

(Prescott et al. 1975, 133, 40: 1-5)

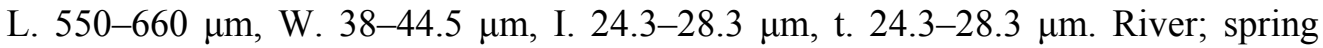
1997; few.

33. Pl. trabecula var. crassum Wittr.

(Pl. 3, Fig. 25) (Prescott et al. 1975, 134, 40: 14)

L. $160 \mu \mathrm{m}$, W. $25 \mu \mathrm{m}$, I. $22.5 \mu \mathrm{m}$, t. $14 \mu \mathrm{m}$. Paddy field; autumn 1997; rare.

34. Pl. trabecula var. elongatum Cedergren

(Pl. 3, Fig. 26; P1. 4, Fig. 36)

(Prescott et al. 1975, 134, 40: 10-11)

L. 162-167 $\mu \mathrm{m}, \mathrm{W} .7-9.6 \mu \mathrm{m}$, I. 5.4-7.8 $\mu \mathrm{m}$, t. 4.8-5.4 $\mu \mathrm{m}$. Lake; winter 1996; common.

35. Pl. trabecula var. maximum (Reinsch) Roll fa. constrictum Scott \& Presc. (Scott and Prescott 1961, 18, 3: 11)

L. $570 \mu \mathrm{m}, \mathrm{W} .40 .5 \mu \mathrm{m}, \mathrm{I} .31 \mu \mathrm{m}, \mathrm{t} .25 .6 \mu \mathrm{m}$. Lake; winter 1996; few.

(P1. 4, Fig. 35)

36. Pl. trabecula fa.

(Pl. 3, Fig. 24)

L. $307-320 \mu \mathrm{m}$, W. $30-31 \mu \mathrm{m}$, I. $26 \mu \mathrm{m}$, t. $17.5-19 \mu \mathrm{m}$; one band at the isthmus. River; spring 1997; very rare.

37. Pl. trochiscum W. \& W. (Prescott et al. 1975, 136, 50: 9-12)

(P1. 3, Fig. 23)

L. $448 \mu \mathrm{m}, \mathrm{W} .32 .4 \mu \mathrm{m}, \mathrm{I} .28 .8 \mu \mathrm{m}$, t. $22.4 \mu \mathrm{m}$. Lake; winter and rainy 1997; rare to few. 


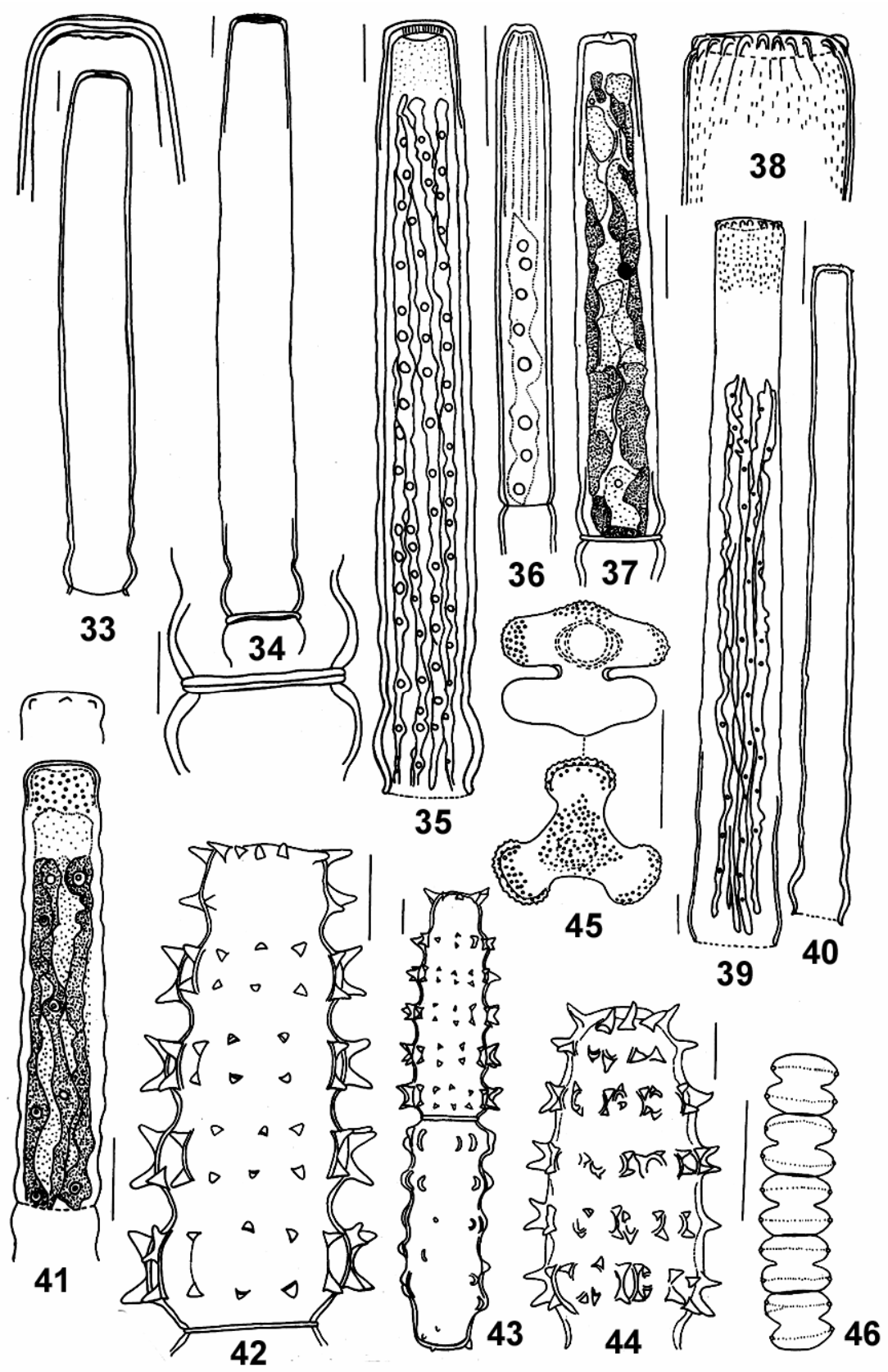

Plate 4

Figs. 33-34. Pleurotaenium trabecula, 35. Pl. trabecula var. maximum fa. constrictum, 36. Pl. trabecula var. elongatum, 37. Pl. ehrenbergii var. chrenbergii, 38-39. Pl. subcoronulatum, 40. Pl. ehrenbergii var. elongatum, 41. Pl. ehrenbergii var. undulatum, 42-43. Pl. kayei, 44. Pl. kayei var. ovoideum, 45. Spondylosium sp., 46. Sphaerozosma aubertianum [Scales $=20 \mu \mathrm{m}$ ]. 
38. * Pl. verrucosum (Bail.) Lund. var. validum Scott and Grönbl.

(Pl. 3, Fig. 22) (Prescott et al. 1975, 139, 51: 1)

L. $275 \mu \mathrm{m}, \mathrm{W} .41 .8-58 \mu \mathrm{m}, \mathrm{I} .29 .7 \mu \mathrm{m}$, t. $23 \mu \mathrm{m}$. River; spring 1997; rare.

Genus: Hyalotheca Ehrenberg ex Ralfs, 1848

39. H. dissiliens (Smith) Bréb. var. hians Wolle

(Pl. 1, Fig. 8)

(Croasdale et al. 1983, 29, 461: 2-3)

L. $12-19 \mu \mathrm{m}$, W. $21.6 \mu \mathrm{m}$, I. $16.2-17.5 \mu \mathrm{m}$. Lake; winter 1997; few.

40. H. dissiliens var. tatrica Racib.

(P1. 1, Figs. 7, 9)

(Croasdale et al. 1983, 29, 461: 4)

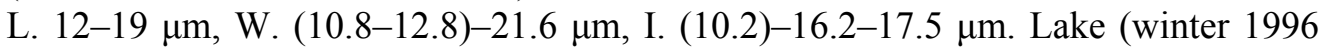
and 1997; rare to common) and river (spring 1997; common).

41. H. mucosa (Mert.) Ehr. ex Ralfs

(Islam and Irfanullah 1999, 122, 1: 3)

Lake (autumn 1997; few) and river (spring 1997; common).

Genus: Groenbladia Teiling, 1952

42. G. neglecta (Racib.) Teiling (Islam and Irfanullah 1998, 93, Figs. 1-3)

Lake (winter 1997) and river (spring 1997); common.

43. G. undulata (Nordst.) Förster

(Islam and Irfanullah 1998, 95, Figs. 4-5)

Lake; autumn 1997; few.

Genus: Bambusina Kützing ex Kützing, 1845

44. B. brebissonii Kütz. var. brebissonii

(P1. 5, Fig. 54)

(Islam 1970, 934, 3: 9-10)

L. 24.3-27 $\mu \mathrm{m}$, W. 16.2-19 $\mu \mathrm{m}$, t. $12 \mu \mathrm{m}$. Lake (winter 1996; rare), river (spring 1997; common) and paddy field (autumn 1997; few).

Genus: Teilingia Bourrelly, 1964

45. T. exigua (Turner) Bourrelly

(Islam and Irfanullah 1999, 122, 1: 11)

Lake; autumn 1997; few.

Genus Sphaerozosma Corda ex Ralfs, 1848

46. *S. aubertianum West, W.

(Croasdale et al. 1983, 2, 448: 1-5)

(Pl. 4, Fig. 46)

L. $10.8 \mu \mathrm{m}, \mathrm{W} .12-13.5 \mu \mathrm{m}, \mathrm{I} .6 .7 \mu \mathrm{m}$; a fine horizontal row of pores across the middle of the semicell; mucilage strand from the pore appearing as granules on the lateral margins. Lake; autumn 1997; common. 


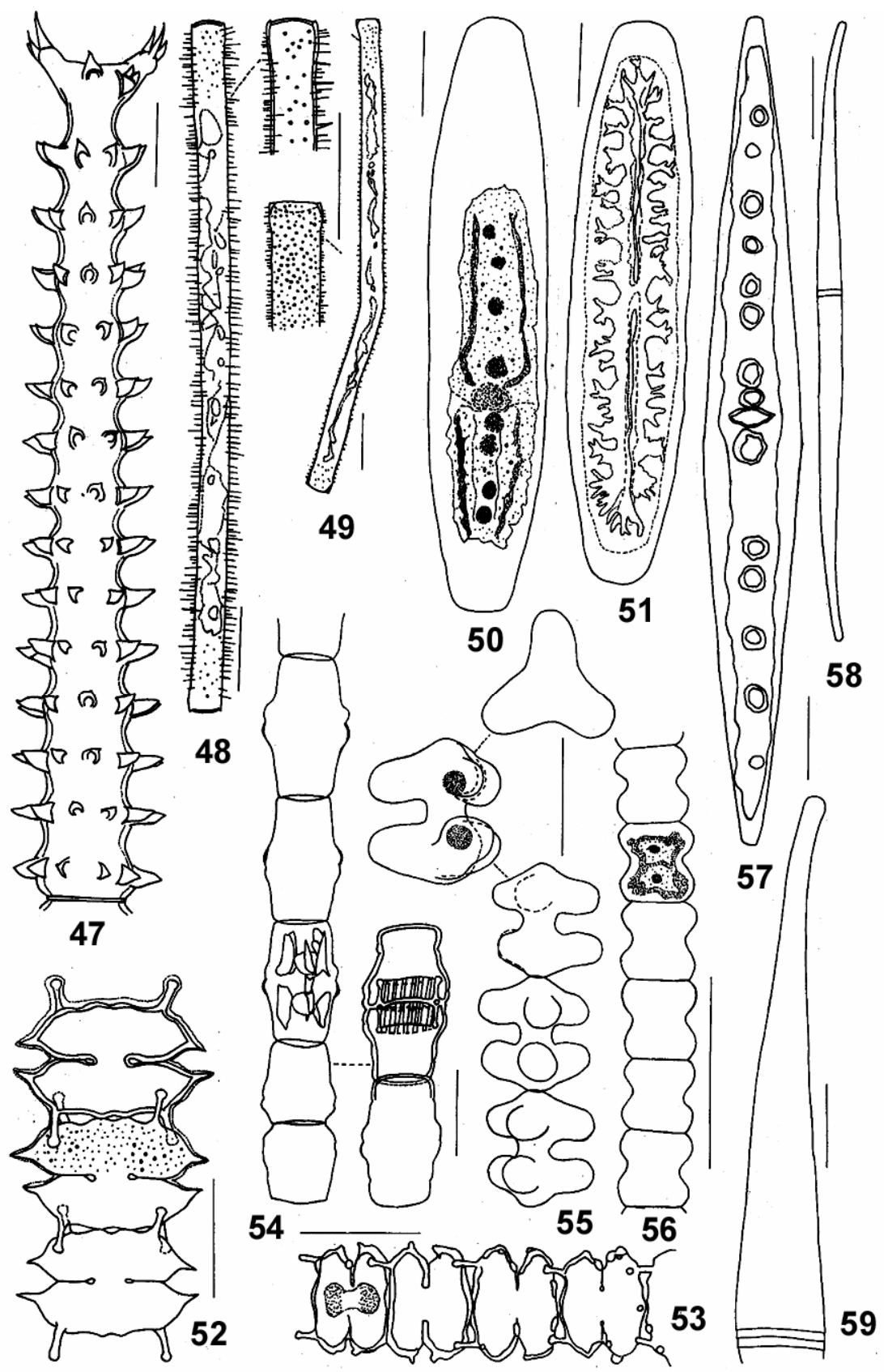

Plate 5

Figs. 47. Triploceras gracile var. undulatum, 48. Gonatozygon aculeatum, 49. G. pilosum, 50-51. Netrium digitus var. lamellosum, 52. Onychonema laeve var. laeve, 53. O. laeve var. micracanthum, 54. Bambusina brebissonii var. brebissonii, 55. Spondylosium javanicum var. javanicum, 56. S. planum var. planum, 57. Closterium var. rectimarginatum, 58. Cl. raflsii var. gracilius, 59. Cl. rostratum var. rostratum [Scales, Figs. $47-51=30 \mu \mathrm{m}$, Figs. 52-59=20 $\mu \mathrm{m}$ ]. 
Genus: Spondylosium Brébisson ex Kützing, 1849

47. S. javanicum (Gutw.) Gronblad var. javanicum

(P1. 5, Fig. 55) (Croasdale et al. 1983, 19, 457: 14-15; Scott and Prescott 1961, 121, 60: 10 as S. nitens (Wall.) Arch. var. triangulare Turner fa. javanicum Gutw.).

L. $27 \mu \mathrm{m}, \mathrm{W} .24 .3 \mu \mathrm{m}$, I. $6.7 \mu \mathrm{m}$. Lake; winter 1996; common.

48. S. panduriforme (Heim.) Teil.

(Islam and Irfanullah 1999, 122, 1: 5-9)

Lake; autumn 1997; common.

49. S. panduriforme var. panduriforme fa. limneticum (W. \& W.) Teil. (Islam and Irfanullah 1999, 122, 1: 10)

Lake; winter 1996 (few) and spring 1997 (common).

50. S. planum var. planum (Wolle) W. \& W.

(Pl. 5, Fig. 56)

(Croasdale et al. 1983, 21, 456: 6-7)

L. $8 \mu \mathrm{m}$, W. $4 \mu \mathrm{m}, \mathrm{I} .2 .7 \mu \mathrm{m}$, t. $2.7 \mu \mathrm{m}$. Paddy field; autumn 1997; few.

51. Spondylosium sp.

(P1. 4, Fig. 45)

L. $24.3-26.3 \mu \mathrm{m}$, W. $28.3-30.4 \mu \mathrm{m}$, I. $7.4 \mu \mathrm{m}$; warts present on the tips of the arms and at the poles. Lake; autumn 1997; rare.

Genus: Onychonema Wallich, 1860

52. O. laeve Nordst. var. laeve

(Pl. 5, Fig. 52)

(Croasdale et al. 1983, 13, 452: 6-10)

L. cpr. 28.3-31 $\mu \mathrm{m}$, L. spr. 18.2-21.6 $\mu \mathrm{m}$, W. csp. 27.7-35.8 $\mu \mathrm{m}$, W. ssp. 21.6-31 $\mu \mathrm{m}$, I. 3-8 $\mu \mathrm{m}$. Lake; winter 1996; rare.

53. O. laeve var. micracanthum Nordst.

(P1. 5, Fig. 53)

(Croasdale et al. 1983, 14, 453: 7-9)

L. $14.8 \mu \mathrm{m}, \mathrm{W}$. csp. $22.3 \mu \mathrm{m}$, I. $6 \mu \mathrm{m}$. Paddy field; autumn 1997; rare.

Genus: Desmidium Agardh, 1848

54. D. aptogonum Bréb. ex Kütz. var. acutius Nordst.

(P1. 1, Figs. 1-2)

(Croasdale et al. 1983, 39, 463: 7-8)

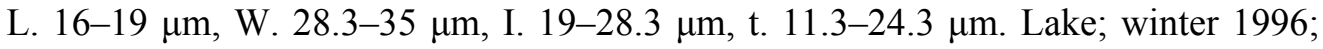
common.

55. D. baileyi (Ralfs) Nordst. var. baileyi

(Pl. 1, Fig. 5)

(Croasdale et al. 1983, 41, 464: 8-9)

L. $21 \mu \mathrm{m}$, W. 21.6-23 $\mu \mathrm{m}$, t. 21.6-23 $\mu \mathrm{m}$; zygospore dimension 27-29.7 $\mu \mathrm{m}$ X 19$21.6 \mu \mathrm{m}$. Lake; winter 1996; common. 


\section{D. baileyi fa. tetragonum Nordst.}

(Islam and Irfanullah 1999, 120, 1: 1)

Lake; winter 1996; few.

57. D. bengalicum Turner

(Pl. 1, Fig. 4)

(Scott and Prescott 1961, 124, 62: 12-13; Islam 1970, 934, 2: 7 \& 7: 6)

L. $19 \mu \mathrm{m}, \mathrm{W} .28 .3-29.7 \mu \mathrm{m}$, Ist. 20.2-21.6 $\mu \mathrm{m}$, t. $12 \mu \mathrm{m}$; zygospore 17.5-19 $\mu \mathrm{m} \times$ $17.5-21 \mu \mathrm{m}$. Colonies are embedded in mucilage with ray-like deposition but no spines. River; winter and spring 1997; few to common.

\section{D. quadrangulatum Ralfs}

(Islam and Irfanullah 1999, 122, 1: 2)

Lake; winter 1996; few.

59. D. swartzii var. ambloydon (Itz.) Rab.

(Islam 1970, 934, 2: 8-9; Croasdale et al. 1983, 49, 468: 5-10)

L. $16.2 \mu \mathrm{m}, \mathrm{W} .35 \mu \mathrm{m}$, I. $29.7-31 \mu \mathrm{m}$, t. $24.3 \mu \mathrm{m}$. Lake; winter 1997; rare.

\section{Acknowledgements}

We are grateful to A.F.M. Badrul Alam, the then Director, Bangladesh Tea Research Institute (BTRI), Srimangal, for providing the logistic, laboratory and other support during this study and also to all his colleagues who extended their help in the laboratory and in supplying necessary information. Thanks also due to James Finley \& Co. for the permission to sample its aquatic habitats.

\section{References}

Croasdale, H.T., Bicudo, C.E.M. and Prescott, G.W. 1983. A Synopsis of North American Desmids. Part II. Desmidiaceae : Placodermae. Sec. 5. The filamentous genera. Univ. Nebraska Press, Lincoln and London.

Islam, A.K.M. Nurul 1970. Contributions to the knowledge of desmids of East Pakistan. Part I. Nova Hedwigia 20: 903-983.

Islam, A.K.M. Nurul 1980. Study on Triplastrum found in Bangladesh with a note on its species. Bangladesh J. Bot. 9(1): 1-12.

Islam, A.K.M. Nurul and Akter, N. 1999. Desmids of Chittagong, Bangladesh. Part 2: Closterium, Docidium, Netrium, Pleurotaenium and Staurastrum. Bangladesh J. Plant Taxon. 6(1): 1930 .

Islam, A.K.M. Nurul and Haroon, A.K.Y. 1980. Desmids of Bangladesh. Int. Revue ges. Hydrobiol. 65(4): 551-604.

Islam, A.K.M. Nurul and Irfanullah, H.M. 1998. New records of three green algal genera for Bangladesh: Desmatractum, Glaucocystis and Groenbladia. Bangladesh J. Plant Taxon. 5(1): 91-95.

Islam, A.K.M. Nurul and Irfanullah, H.M. 1999. New records of desmids for Bangladesh. II. Thirteen taxa. Bangladesh J. Bot. 28(2): 117-123. 
Islam, A.K.M. Nurul and Irfanullah, H.M. 2000. Hydrobiological studies within the tea gardens at Srimangal, Bangladesh. I. Aquatic macrophytes. Bangladesh J. Plant Taxon. 7(1): 29-42.

Islam, A.K.M. Nurul and Irfanullah, H.M. 2005a. Hydrobiological studies within the tea gardens at Srimangal, Bangladesh. II. Algal flora (excluding Chlorophyceae). Bangladesh J. Plant Taxon. 12(1): 33-52.

Islam, A.K.M. Nurul and Irfanullah, H.M. 2005b. Hydrobiological studies within the tea gardens at Srimangal, Bangladesh. III. Chlorophyceae (excluding desmids). Bangladesh J. Plant Taxon. 12(2): 19-37.

Prescott, G.W., Croasdale, H.T. and Vinyard, W.C. 1975. A synopsis of North American desmids. Part II. Desmidiaceae: Placodermae. Sec I. Univ. Nebraska Press, Lincoln, pp. 275.

Růžička, J. 1977. Die Desmidiaceen Mitteleuropas. Band 1; 1 Lief. E. Schw. Verlagsb., Stuttgart, pp. $291+$ pls. $1-44$.

Scott, A.M. and Prescott, G.W. 1958. Some freshwater algae from Arnhem Land in the Northern Territory of Australia. Rec. American-Australian Sci. Expn. to Arnhem Land (Part 2) 3: 8-136.

Scott, A.M. and Prescott, G.W. 1961. Indonesian Desmids. Hydrobiologia 17(1-2): 1-132 + pls. 63 .

Smith, G.M. 1924. Phytoplankton of the Inland Lakes of Wisconsin. Part II. Desmidiaceae. Wisconsin Geol. \& Nat. Hist. Surv. Bull. 57(Part II): 1-227, pls. 52-88 + text figs. 1-17. 